\title{
DIAGNÓSTICO Y TRATAMIENTO OPORTUNOS EN LA PREVENCIÓN DE COMPLICACIONES CARDIOVASCULADORES DE LA ENFERMEDAD DE KAWASAKI, HOSPITAL DE NIÑOS ROBERTO GILBERT ELIZALDE, ENERO 2005 HASTA DICIEMBRE 2011
} TIMELY DIAGNOSIS AND TREATMENT IN THE PREVENTION OF CARDIOVASCULAR COMPLICATIONS OF KAWASAKI DISEASE, IN ROBERTO GILBERT ELIZALDE CHILDREN'S HOSPITAL, FROM JANUARY 2005 TO DECEMBER 2011

\author{
DIAGNÓSTICO ETRATAMENTO ATUALMENTENA PREVENÇĀO DECOMPLICAÇŌES CARDIOVASCULATÓRIAS DA DOENÇA
} DEKAWASAKI, HOSPITALINFANTIL DE ROBERTO GILBERT ELIZALDE, JANEIRO DE2005 A DEZEMBRO DE2011

\author{
MARÍA ALEJANDRA ARMIJOS VILLACÍS', MARÍA VERÓNICA CASTILLO HIGGINS', ANDREA PATIÑO ARMIJOS', ISABEL MARÍA \\ SALCEDO VELARDE ${ }^{2}$ \\ 1 Universidad Católica de Santiago de Guayaquil; Guayaquil, Ecuador \\ 2 Hospital de niños Roberto Gilbert Elizalde; Guayaquil, Ecuador
}

\begin{abstract}
Introducción: la enfermedad de Kawasaki es una arteritis de vasos de pequeñoy mediano calibre, la cual afecta en su gran mayoría a pacientes pediátricos y se considera como causa principal de cardiopatía adquirida en niños. Objetivo: relacionar el tipo de tratamiento (oportuno vs. no oportuno) con la presentación de complicaciones cardiovasculares en pacientes diagnosticados con enfermedad de Kawasaki. Métodos: estudio observacional, analítico, transversal, retrospectivo, donde se revisaron las historias clínicas de los pacientes con diagnóstico de enfermedad de Kawasaki en el hospital de niños Roberto Gilbert Elizalde, desde enero de 2005 a diciembre de 2011. Los criterios de inclusión fueron pacientes con diagnóstico de enfermedad de Kawasaki, edad entre 0 y 17 años y que cuenten con informes ecocardiográficos. Se excluyeron pacientes con cardiopatía previa, ya sea congénita 0 adquirida, que hayan presentado otros posibles focos infecciosos y también aquellos con historias clínicas incompletas. Resultados: la muestra total de pacientes que cumplieron los criterios de inclusión fue de 42 . De ellos, 29 pacientes (69\%) fueron de sexo masculino y el promedio de edad fue de 1.94 años. Según la combinación del tiempo de diagnóstico y tratamiento dado se dividió a los pacientes en dos grupos. El grupo de tratamiento oportuno, grupo 1 fue de 19 pacientes (45.2\%), yel grupo de tratamiento no oportuno, grupo 2, fue de 23 pacientes (54.7\%). En el Grupo 2 se encontró una diferencia estadísticamente significativa en el número total de pacientes que presentaron cualquier tipo de complicaciones cardiovasculares $(\mathrm{p}<0.001)$, pacientes con dilatación aneurismática $(\mathrm{p} 0.001)$, y con derrame pericárdico ( $\mathrm{p} 0.002)$. Conclusión: las complicaciones cardiovasculares de la enfermedad de Kawasakiestán relacionadas al diagnósticoy tratamiento inoportunos, tardíos e inapropiados, por lo que sonnecesarios mayores esfuerzos educativos acerca de la enfermedad y su detección para el personal médicoy padres o cuidadores primarios.
\end{abstract}

PALABRAS CLAVE: síndrome mucocutáneo linfonodular, enfermedades cardiovasculares, enfermedades pediátricas.

Abstract

Introduction: Kawasakidisease is a small and medium caliber vessel arteritis, which mostly affects pediatric patients and is considered the main cause of acquired heart disease in children. Objective: to compare the type of treatment (timely vs. untimely) with the presentation of cardiovascular complications in patients diagnosed with Kawasaki disease. Methods: observational, analytical, cross-sectional, retrospective study, where the medical records of patients, with a diagnosis of Kawasaki disease, were reviewed, at the Roberto Gilbert Elizalde children's hospital, from January 2005 to December 2011 . The inclusion criteria were patients diagnosed with Kawasaki disease, age between 0 and 17 years, and with echocardiographic reports. Patients with previous heart disease, whether congenital or acquired, who had other possible infectious foci and those with incomplete medical records were excluded. Results: the total sample of patients that fulfill the inclusion criteria was 42 . Of this sample, 29 patients (69\%) were male and the average age was 1.94 years. Depending on the combination of diagnosis and treatment time given, patients were divided into two groups. The first group was composed of a total of 19 (45.2\%) patients, that is the timely treatment group, the second group with a total of 23 patients (54.7\%) is the non-timely treatment group. The second group was found a statistically significant difference in the total number of patients who presented any type of cardiovascular complications $(p<0.001)$, patients with aneurysmal dilation ( $p 0.001)$, and with pericardial effusion ( $\mathrm{p}$ 0.002). Conclusion: Cardiovascular complications of Kawasaki disease are related to untimely, late and inappropriate diagnosis and treatment, therefore greater educational efforts about the disease and its detection are necessary for medical personnel and parents or primary caregivers.

KEYWORDS: mucocutaneous lymphnode syndrome, cardiovascular diseases, enfermedades pediátricas.

Resumo

Introdução: a doença de Kawasaki é uma arterite de pequenos e médios vasos, que afeta principalmente pacientes pediátricos e é considerada a principal causa de doença cardíaca adquirida em crianças. Objetivo: relacionar o tipo de tratamento (oportuno versus prematuro) com a apresentação de complicações cardiovasculares em pacientes com diagnóstico de doença de Kawasaki. Métodos: estudo observacional, analítico, transversal, retrospectivo, no qual foram revisados os prontuários de pacientes com diagnóstico de doença de Kawasakino hospital infantil Roberto Gilbert Elizalde, no período de janeiro de 2005 a dezembro de 2011. Os critérios de inclusão foram: pacientes com diagnóstico de doença de Kawasaki, idade entre 0 e 17 anos e que possuem laudos ecocardiográficos. Foram excluídos pacientes com cardiopatia prévia, congênita ou adquirida, que apresentaram outros possíveis focos infecciosos e também aqueles com prontuários médicos incompletos. Resultados: a amostra total de pacientes que atendeu aos critérios de inclusão foi de 42. Desses, 29 pacientes (69\%)eram do sexo masculino e a médiade idade foi de 1,94 anos. Dependendo da combinação de diagnósticoe tempo de tratamento, os pacientes foram divididos em dois grupos. 0 grupo de tratamento oportuno, grupo 1, foi de 19 pacientes (45,2\%), e o grupo de tratamento não oportuno, grupo 2 , foi de 23 pacientes $(54,7 \%)$. No Grupo 2 , foi encontrada diferença estatisticamente significante no número total de pacientes que apresentaram algum tipo de complicação cardiovascular $(\mathrm{p}<0,001)$, pacientes com dilatação aneurismática ( $\mathrm{p} 0,001)$ e com derrame pericárdico $(\mathrm{p} 0,002)$. Conclusão: as complicações cardiovasculares da doença de Kawasaki estão relacionadas a diagnósticos e tratamentos inadequados, tardios e inadequados, portanto, maiores esforços educacionais sobre a doença e sua detecção são necessários para 0 pessoal médico o os pais ou cuidadores primários.

PALAVRAS-CHAVE: síndrome de linfonodos mucocutâneo, doenças cardiovasculares, doenças pediátricas. 
INTRODUCCIÓN

La enfermedad de Kawasaki (EK), o síndrome linfonodular mucocutáneo, fue descrita por primera vez en 1967 por el pediatra japonés Tomisaku Kawasaki. Se define como una arteritis que afecta a vasos de pequeño y mediano calibre, multisistémica, febril, aguda, de etiología desconocida, la cual afecta en su gran mayoría a pacientes pediátricos ${ }^{1}$. Tiene una distribución etaria máxima entre los 12 y 24 meses de edad; el $50 \%$ de los pacientes son menores de 2 años, el $80 \%$ menores de 4 años, y rara vez aparece la enfermedad en mayores de 8 años ${ }^{2}$. Es más frecuente en el sexo masculino con una proporción hombre:mujer de 1,5:13.

Sus signos y síntomas son inespecíficos, lo que dificulta un diagnóstico precoz y conlleva frecuentemente a complicaciones4. Por la predilección de la EK a la formación de aneurismas y cambios histopatológicos en la circulación coronaria, se considera como causa principal de cardiopatía adquirida en niños ${ }^{1,5,6}$, destacando entre sus complicaciones los aneurismas coronarios, cardiopatía isquémica, insuficiencia mitral, insuficiencia aórtica, derrame pericárdico y pericarditis ${ }^{7}$. Se coincide que el diagnóstico tardío y el tratamiento inadecuado son factores desencadenantes y altamente predictivos de dichas complicaciones ${ }^{8}$, las mismas que pueden llevar al infarto agudo de miocardio e incluso a la muerte súbita ${ }^{5}$.

En la actualidad no existen estadísticas nacionales ni locales de importancia respecto a esta patología, lo cual podría resultar en que muchos casos sean obviados o erróneamente diagnosticados con otro tipo de enfermedad exantemática 9 . El presente estudio analiza la relación entre el diagnóstico y tratamiento oportunos y la presentación de las complicaciones en los pacientes con EK.

METODOLOGÍA

Estudio observacional, analítico, transversal, retrospectivo; se revisaron las historias clínicas de los pacientes con diagnóstico de enfermedad de Kawasaki en el hospital de niños Roberto Gilbert Elizalde, desdeel mes de enero de 2005 a diciembre de 2011. La recolección de la información se realizó consultando la base de datos de dicho hospital incluyendo en la búsqueda todos los pacientes ingresados bajo el código M30.3, de la décima versión del Sistema Internacional de Clasificación de Enfermedades CIE10. Como resultado de la búsqueda se obtuvo 48 pacientes distribuidos de la siguiente forma: 3, en el 2007; 16, en el 2008; 10, en el 2009; 9 , en el 2010 y 10, en el 2011.

Los criterios de inclusión fueron: pacientes con diagnóstico de enfermedad de Kawasaki, edad entre 0 y 17 años, existencia de los respectivos informes ecocardiográficos. Se excluyeron a los pacientes con cardiopatía previa, ya sea congénita o adquirida, que hayan presentado otros posibles focos infecciosos (faringoamigdalitis estreptocócica, exantema característico de sarampión, diagnóstico de escarlatina, y otros pacientes que se haya identificado por serología como: mononucleosis, adenovirus o leptospirosis), y pacientes con historias clínicas incompletas.

\section{VARIABLES}

Los datos o variables analizados fueron: edad (años), sexo, procedencia (provincia), el tiempo de inicio de fiebre hasta el ingreso (días), tiempo de inicio de fiebre hasta el diagnóstico (días), tiempo de inicio de fiebre previo al tratamiento (días), exantema polimorfo, inyección conjuntival, cambios en la mucosa oral, alteraciones en las extremidades y linfadenopatía cervical. Además se registró la fórmula leucocitaria, plaquetas, alteración ecocardiográfica, complicaciones cardiológicas, especificando el tiempo transcurrido hasta su aparición, el tipo de tratamiento, dosis, duración. Para la aplicación y análisis se consideró como tratamiento oportuno aquel que se lleve a cabo dentro de los primeros 10 días de fiebre administrando los siguientes fármacos: ácido acetilsalicílico por vía oral en dosis de 80 a 100 $\mathrm{mg} / \mathrm{kg} /$ día y gammaglobulina por vía intravenosa en dosis única de $2 \mathrm{~g} / \mathrm{kg}$.

\section{ANÁLISIS ESTADÍSTICO}

El objetivo primario del presente estudio es relacionar el tipo de tratamiento (oportuno vs. no oportuno) con la presentación de complicaciones cardiovasculares en pacientes diagnosticados con EK. Las diferentes variables obtenidas fueron ingresadas de manera inmediata a una base de datos creada en una hoja de cálculo de Microsoft Excel 7.0 para Windows Vista. Para su análisis estadístico respectivo, se obtuvo el promedio y la desviación estándar de las variables cuantitativas continuas; para obtener el valor p de las mismas se utilizó la prueba de T student. Para las variables cualitativas, se utilizó la prueba de chi cuadrado, así mismo para obtener el valor p correspondiente. Para el análisis de las variables se utilizó Microsoft Excel 7.0 y Epiinfo 3.5.4. En este estudio, se utilizó el método de muestro no aleatorio. 
RESULTADOS

La muestra total de pacientes que cumplieron los criterios de inclusión fue de 42. De ellos, 29 pacientes (69\%) fueron de sexo masculino y el promedio de edad fue de 1.94 años.

Según la combinación del tiempo de diagnostico y tratamiento dado, se dividió a los pacientes en dos grupos: en el grupo 1, de pacientes con tratamiento oportuno (aplicado hasta los primeros 10 días de fiebre administrando ácido acetilsalicílico $80-100 \mathrm{mg} / \mathrm{kg} /$ día y dosis única de gammaglobulina por vía intravenosa de $2 \mathrm{~g} / \mathrm{kg}$ ), se registraron 19 pacientes (45.2\%); y en el grupo 2 de pacientes con tratamiento no oportuno (tratamiento aplicado después de los 10 primeros días de fiebre o que no recibieron los fármacos y dosis adecuadas), se encontraron 23 pacientes (54.7\%), tabla 1.

\section{TABLA 1. CARACTERÍSTICAS BASALES*}

\begin{tabular}{|c|c|c|c|}
\hline & $\begin{array}{r}\text { GRUPO } 1 \\
\text { TXA OPORTUNO } \mathrm{K}=19\end{array}$ & $\begin{array}{r}\text { GRUPO } 2 \\
\text { TX NO PORTUNO } N=23\end{array}$ & $\begin{array}{r}\text { VALOR } \\
P\end{array}$ \\
\hline Edad & $2.29 \pm 2.11$ & $1.64 \pm 1.28$ & 0.25 \\
\hline Sexo masculino & $14(73.6)$ & $15(65.2)$ & 0.55 \\
\hline \multicolumn{4}{|l|}{ Procedencia } \\
\hline Guayas & 12 (63.1) & 17 (73.9) & 0.45 \\
\hline Manabí & $4(21)$ & $2(8.6)$ & 0.25 \\
\hline Los Ríos & $1(5)$ & $1(4.3)$ & 0.88 \\
\hline Azuay & $0(0)$ & $2(8.6)$ & 0.18 \\
\hline El Oro & $1(5)$ & $1(4.3)$ & 0.88 \\
\hline Pichincha & $1(5)$ & $0(0)$ & 0.26 \\
\hline \multicolumn{4}{|l|}{ Síntomas ${ }^{b}$} \\
\hline Fiebre & $19(100)$ & $22(95.6)$ & 0.35 \\
\hline Iny. conjuntival ${ }^{\mathrm{C}}$ & $8(42.1)$ & $11(47.8)$ & 0.71 \\
\hline Exantema & $17(89.4)$ & $21(91.3)$ & 0.84 \\
\hline Cambios mucosa oral & $15(78.9)$ & 18 (78.2) & 0.95 \\
\hline Alt.extremidades ${ }^{d}$ & $13(68.4)$ & $19(82.6)$ & 0.28 \\
\hline Linfadenopatía cerv. $^{e}$ & $10(52.6)$ & $8(34.7)$ & 0.24 \\
\hline Fiebre/ingreso ${ }^{f}$ & $6.05 \pm 1.35$ & $13.9 \pm 4$ & $<0.05$ \\
\hline Fiebre/diagnóstico ${ }^{9}$ & $6.84 \pm 1.74$ & $10.6 \pm 7.8$ & $<0.05$ \\
\hline \multicolumn{4}{|l|}{ Exámenes } \\
\hline GBh & $23088 \pm 20089$ & $15973 \pm 6923.2$ & 0.15 \\
\hline Neutrófilos(\%) & $62.5 \pm 24$ & $60.8 \pm 17.1$ & 0.79 \\
\hline Linfocitos(\%) & $27.4 \pm 20.9$ & $28.2 \pm 14.7$ & 0.90 \\
\hline Plaquetas & $386631.5 \pm 158619.1$ & $504391.3 \pm 210830.7$ & 0.05 \\
\hline Alt. ecocardiográficasi & $5(26.3)$ & $21(91.3)$ & $<0.05$ \\
\hline
\end{tabular}

*Valores en paréntesis corresponden a los porcentajes de cada grupo; valores \pm corresponden a desviación. ${ }^{\mathrm{a}} \mathrm{T} \mathrm{X}=$ tratamiento; ${ }^{\mathrm{b}}$ Síntomas establecidos según el Tercer Simposio de Tokio en 1988 para la Enfermedad de Kawasaki y las recomendaciones de 2004 de la Academia Americana de Pediatría (AAP); ' inyección conjuntival; ${ }^{\mathrm{C}}$ alteraciones en extremidades; ${ }^{\mathrm{e}}$ linfadenopatía cervical; ${ }^{\mathrm{f}}$ Tiempo entre inicio de fiebre e ingreso a unidad hospitalaria; ${ }^{9}$ Tiempo entre inicio de fiebre y diagnóstico de enfermedad de Kawasaki; ' $\mathrm{GB}$ = glóbulos blancos; ; alteraciones ecográficas

Entre las variables basales estudiadas se encontró una diferencia estadísticamente significativa en el tiempo transcurrido desde el inicio de la fiebre hasta el ingreso del paciente a la unidad hospitalaria ( $p<0.05$ ), al igual que en el tiempo entre el inicio de la fiebre y el diagnóstico de la enferme- dad ( $\mathrm{p}<0.05)$. Entre los exámenes recolectados, se hallaron diferencias significativas en el conteo plaquetario ( $\mathrm{p} 0.04$ ) y el número de pacientes con alteraciones ecocardiográficas ( $\mathrm{p}<0.05)$.

COMPLICACIONES

En la tabla 2 se registraron las complicaciones presentadas por los pacientes en cada uno de los grupos. Se encontró una diferencia estadísticamente significativa en el número total de pacientes que presentaron cualquier tipo de complicaciones cardiovasculares $(\mathrm{p}<0.001)$, pacientes con dilatación aneurismática ( $\mathrm{p} 0.001)$, y con derrame pericárdico ( $\mathrm{p} 0.002$ ).

\begin{tabular}{|c|c|c|c|}
\hline & $\begin{array}{r}\text { GRUPO TXA } \\
\text { ORTUNO }=19\end{array}$ & $\begin{array}{r}\text { GRUPO TX NO } \\
\text { OPORTUNO N=23 }\end{array}$ & VALOR P \\
\hline Complicaciones cardiovasculares & $5 \quad 4(21)$ & $18(78.2)$ & $<0.001$ \\
\hline Dilt. aneurismática ${ }^{b}$ & $2(10.5)$ & $13(56.5)$ & 0.001 \\
\hline Derrame pericárdico & $1(5)$ & $11(47.8)$ & 0.002 \\
\hline Insuficiencia mitral & $0(0)$ & $1(4.3)$ & 0.35 \\
\hline Otras ${ }^{\complement}$ & $1(5)$ & $1(4.3)$ & 0.88 \\
\hline Otras complicaciones & $3(15.8)$ & $2(8.6)$ & 0.47 \\
\hline Reingreso & $1(5)$ & $0(0)$ & 0.26 \\
\hline
\end{tabular}

*Valores representan el número de pacientes que presentaron dicha variable; valores en paréntesis corresponden a los porcentajes de cada grupo.

${ }^{a} \mathrm{Tx}=$ tratamiento; ' Dilatación aneurismática; 'Otras (pericarditis, vasculitis severa coronaria).

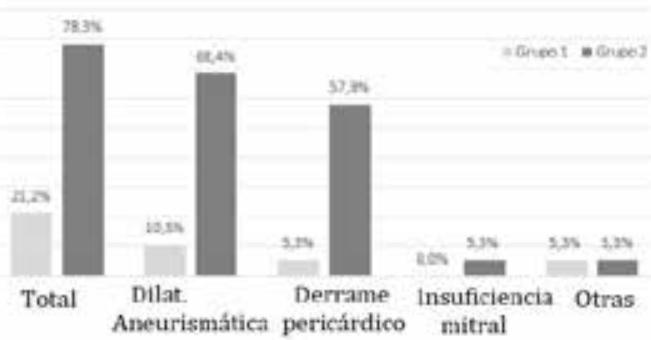

Figura 1. complicaciones cardiovasculares, complicaciones presentadas en cada grupo.

\section{DISCUSIÓN}

El diagnóstico de la enfermedad de Kawasaki después de 10 días del inicio de los síntomas, no es un evento aislado sino más bien común ${ }^{10-12}$. En la tabla 1, podemos observar que el tiempo de inicio de fiebre hasta el ingreso de los pacientes del grupo 2 fue muy prolongado en comparación con los del grupo 1 (6.05 días vs. 13.9 días; $\mathrm{p}<0.05$ ). Del mismo modo lo fue el tiempo de inicio de fiebre y el diagnóstico de Enfermedad de Kawasaki (6.8 días vs. 10.6 días; $\mathrm{p}<0.05)$. El retraso inicial del reconocimiento adecuado de la enfermedad por parte de padres y personal médico, tiene serias consecuencias en el pronóstico del paciente. Luca et al realizaron un perfil comprensivo de los pacientes en riesgo de diagnóstico tardío de EK, incluyendo variables no relacionadas al paciente como el comportamiento de sus cuidadores 
primarios y la experiencia del personal médico; llegaron a la conclusión de que es necesario y urgente realizar intervenciones de preparación en ambos grupos humanos para evitar el diagnóstico tardío $^{13}$. Otra posibilidad, como lo plantea Anderson et al., es que el diagnóstico después del décimo día no se debe a lo mencionado anteriormente sino que los síntomas iniciales de este grupo de diagnóstico tardío, están dispersos en el tiempo; opuesto a la superposición de síntomas en los pacientes diagnosticados tempranamente ${ }^{14}$.

Al analizar los exámenes delaboratorio realizados, se observa una diferencia significativa en el conteo plaquetario ( $\mathrm{p} 0.04$ ), que es parte de la historia natural de la enfermedad. La trombocitosis aparece habitualmente en la segunda semana de la enfermedad, llegando a su pico en la tercera semana. ${ }^{9}$ En el grupo 2 del estudio, su ingreso y diagnóstico tardío los ha llevado a alcanzar una etapa más avanzada de la enfermedad, lo que corresponde a la trombocitosis registrada, que en el grupo 1 no se observa de manera tan marcada.

En la fisiopatología de la enfermedad de Kawasa$\mathrm{ki}$, se conoce que los cambios arteriales coronarios usualmente se inician a partir de los días 7 y 10 de la enfermedad ${ }^{15}$. Al inicio dichos cambios son imperceptibles como alteraciones ecocardiográficas; pero al aumentar los días, los cambios son más acentuados y detectables. En el grupo 2, se encontraron 21 pacientes $(91.3 \%$; $\mathrm{p}<0.05)$ con alteraciones ecocardiográficas, hallazgo que coincide con lo descrito en la literatura debido al retraso en el ingreso del paciente y su respectivo diagnóstico.

Las complicaciones, principalmente cardiovasculares, fueron significativamente más frecuentes en el grupo 2 (4 vs. 18; $p<0.001$ ), específicamente la dilatación aneurismática ( $\mathrm{p} 0.001$ ) y el derrame pericárdico ( $\mathrm{p}$ 0.002). Estos hallazgos, son resultado de una combinación de factores. El tratamiento óptimo de la enfermedad de Kawasaki debe iniciarse dentro de los 7 primeros días de la enfermedad, tiempo que se ha extendido hasta un máximo de 10 dias $^{16-18}$. Esto se debe a que las lesiones arteriales que presentan los pacientes con EK tienen una progresión característica según la duración de la misma ${ }^{19}$, y el tratamiento tiene como finalidad la prevención de estas lesiones. En el caso de los pacientes del grupo 2, muchos fueron ingresados con esas lesiones probablemente ya en desarrollo, aunque indetectables en un principio. Marasini et al reportaron que la efectividad de la IgG intravenosa después del décimo día de enfer- medad, disminuye ${ }^{20}$. Debido a la complejidad éti$\mathrm{ca}$, no existen estudios claros de la efectividad de la IgG intravenosa vs. placebo después del décimo día. A pesar de ésto, se recomienda la utilización de la IgG intravenosa en pacientes pediátricos después del décimo día de enfermedad con fiebre persistente o aneurismas coronarios y evidencia de inflamación sistémica ${ }^{16}$. Muta et al, concluyeron que el tratamiento con IgC intravenosa después de los 10 días del inicio de la patología se considera efectivo para disminuir la inflamación sistémica y es suficiente para prevenir lesiones coronarias ${ }^{21}$. En el grupo 2, cinco pacientes a pesar del diagnóstico tardío, no recibieron IgG intravenosa y de ellos, 4 pacientes $(80 \%)$ presentaron complicaciones cardiovasculares. La combinación de un tratamiento inadecuado y tardío, mostró como resultado en el presente estudio una mayor frecuencia de complicaciones cardiovasculares. Similares resultados fueron obtenidos de Wilder et al. y Juan et al., donde se detectó un riesgo aumentado de desarrollar aneurismas arteriales coronarios y lesiones arteriales coronarias, respectivamente, asociado a la demora diagnóstica ${ }^{22,23}$.

El presente estudio tiene algunas limitaciones. Primero, fueron incluidos todos los pacientes que cumplieron con los criterios de inclusión sin utilizar un método de muestreo aleatorio. Segundo, cada uno de los pacientes fueron evaluados y diagnosticados por personal médico distinto, y a pesar de que existen criterios para su diagnóstico, muchos de estos son sujetos a la subjetividad (exantema polimorfo, inyección conjuntival, cambios en la mucosa oral, alteraciones en las extremidades) y experiencia del personal. Tercero, la información respecto al inicio de la fiebre puede estar influenciada por el estado psicológico, nivel de educación, edad, etc. a quien se pide dicha información; por lo general padres o cuidadores primarios. Cuarto, no se reportó datos de laboratorio importantes como alanino aminotransferasa, bilirrubina total, sodio sérico, VSG y PCR, factores importantes para la detección de inflamación sistémica y falla inicial del tratamiento con IgC intravenosa ${ }^{24-26}$.

Por último, en este estudio no se dividió al grupo de pacientes con tratamiento no oportuno en un grupo de quienes si recibieron el tratamiento correcto pero de manera tardía, y otro con quienes nunca recibieron el tratamiento adecuado. Esta división nos hubiera podido dar un enfoque más claro de cuáles son las consecuencias del diagnóstico tardío y la efectividad del tratamiento. 
CONCLUSIÓN

Las complicaciones cardiovasculares dela enfermedad de Kawasaki están relacionadas al diagnóstico y tratamiento inoportuno, tardío e inapropiado, por lo que son necesarios mayores esfuerzos educativos acerca de la enfermedad y su detección para el personal médico y padres o cuidadores primarios.

\section{REFERENCIAS BIBLIOGRÁFICAS}

1. Jiménez, D. G., Canabal, L. A., Treviño, S. J., Ramos, E., \& García, C. B. Enfermedad de Kawasaki. Experiencia en nuestro hospital. Bol pediatr 2010; 50: 4-10.

2. Huang W-C, Huang L-M, Chang I-Setal. Epidemiologic features of Kawasaki disease in Taiwan, 2003-2006. Pediatrics 2009; 123: e401-5.

3. Son MF, Gauvreau K, Ma L, Baker AL, Sundel RP, Fulton DR, etal. Diagnosis and treatment of Kawasaki Disease: analysis of 27 US pediatric hospitals from 2001-2006. Pediatrics. 2009;124:1-8

4. Prieto, L. M., Cuesta, M. T., Guillén, S., Ruiz, M., Cortés, M. T., Rubio, B., \& Ramos, J. T. . Enfermedad de Kawasaki: diagnóstico y tratamiento. Anales de Pediatría 2010; vol.73, No. 1, pp. 25-30).

5. Baquero, R., Tuesca, R., Muñoz, C., Pérez, J., Molina, T., \& Bustamante, M. C. Enfermedad de Kawasaki en niños hospitalizados en cinco centros de Barranquilla, Colombia, 2002-2008. Infectio, 2011, vol. 14, no 2.

6. Bradley DJ, Glode MP. Kawasaki disease. The mystery continues. West JMed 1998; 168:23-29.

7. Sepulveda, Diana Marcela Ortiz; de grado, trabajo; de ciencias, Facultad. Enfermedad de Kawasaki: serie de casos enero 2006-diciembre 2008 en Bogota.

8. Fernanda, F., Serena, C., \& Donato, R. Kawasaki syndrome: an intriguing disease with numerous unsolved dilemmas. Pediatric Rheumatology 2010;9.

9. Cardozo-López, Margarita, etal. Enfermedad de Kawasaki: diagnóstico y tratamiento. Revista CES Medicina Volumen, 2012, vol. 26, no 2.

10. Minich LLA, Sleeper LA, Atz AM, et al; Pediatric Heart Network Investigators. Delayed diagnosis of Kawasaki disease: what are the risk factors? Pediatrics. 2007;120(6).

11. DuZD, DiZ, DuJB, etal. Comparison of efficacy among early, conventional and late intravenous gamma globulin treatment of Kawasaki disease [in Chinese with English abstract]. Zhonghua Yi Xue Za Zhi. 2009;89 (26):1841-1843.

12. Sittiwangkul R, Pongprot Y, Silvilairat S, Phornphutkul C. Delayed diagnosis of Kawasaki disease: risk factors and outcome of treatment. Ann Trop Paediatr. 2011;31(2):109-114.

13. Luca, N., Ching, J. C., Manlhiot, C., McCrindle, B. W., \& Yeung, R.S Predictors of delayed treatment of Kawasaki disease in community and tertiary care hospitals. Pediatric Rheumatology Online Journal, 2012;10 (1): A89.
14. Anderson MS, Todd JK, Glodé MP. Delayed diagnosis of Kawasaki syndrome: an analysis of the problem. Pediatrics. 2005;115(4).

15. Baer AZ, Rubin LG, Shapiro CA, et al. Prevalence of coronary artery lesions on the initial echocardiogram in Kawasaki syndrome. Arch Pediatr Adolesc Med. 2006;160 (7):686-690.

16. Newburger JW, Takahashi $M$, Gerber MA, et al; Committee on Rheumatic Fever, Endocarditis and Kawasaki Disease; ; Council on Cardiovascular Disease in the Young; ; American Heart Association; American Academy of Pediatrics. Diagnosis, treatment, and longterm management of Kawasaki disease: a statement for health professionals from the Committee on Rheumatic Fever, Endocarditis and Kawasaki disease, Council on Cardiovascular Disease in the Young, American Heart Association. Circulation. 2004;110(17):2747-2771.

17. Zhang T, Yanagawa H, Oki I, et al. Factors related to cardiac sequelae of Kawasaki disease. Eur J Pediatr. 1999;158(9):694-697.

18. Yanagawa H, TuohongZ, Oki I, etal. Effects of gammaglobulin on the cardiac sequelae of Kawasaki disease. Pediatr Cardiol. 1999; 20(4):248-251.

19. Burns JC, Kushner HI, Bastian JF, Shike H, Shimizu C, Matsubara T, TurnerCL, etal. Kawasaki disease: A brief history. Pediatrics 2000; 106: E27.

20. Marasini M, Pongiglione G, Gazzolo D, Campelli A, Ribaldone D, Caponnetto S. Late intravenous gamma globulin treatment in infants and children with Kawasaki disease and coronary artery abnormalities. Am J Cardiol. 1991;68(8):796-797.

21. Muta, H., Ishii, M., Yashiro, M., Uehara, R., \& Nakamura, Y. Lateintravenous immunoglobulin treatment in patients with Kawasaki disease. Pediatrics 2012, 129(2), e291-e297.

22. Wilder MS, Palinkas LA, Kao AS, BastianJF, Turner CL, Burns JC. Delayed diagnosis by physicians contributes to the development of coronary artery aneurysms in children with Kawasaki syndrome. Pediatr Infect Dis J. 2007;26(3):256-260.

23. Juan CC, Hwang B, Lee PC, etal. The clinical manifestations and risk factors of adelayed diagnosis of Kawasaki disease. JChin Med Assoc. 2007;70(9):374-379.

24. EgamiK, Muta H, Ishii M, etal. Prediction of resistance to intravenous immunoglobulin treatment in patients with Kawasaki disease. J Pediatr. 2006;149(2):237-240

25. Kobayashi $\mathrm{T}$, Inoue $\mathrm{Y}$, Takeuchi $\mathrm{K}$, et al. Prediction of intravenous immunoglobulin unresponsiveness in patients with Kawasaki disease. Circulation. 2006;113(22): 2606-2612.

26. SanoT, KurotobiS, Matsuzaki K, etal. Prediction of nonresponsiveness to standard high-dose gamma-globulin therapy in patients with acute Kawasaki disease before starting initial treatment. Eur J Pediatr. 2007;166(2): 131-137 